\title{
CONDITION MONITORING OF OPERATING PIPELINES WITH OPERATIONAL MODAL ANALYSIS APPLICATION
}

\author{
Aleksey Mironov ${ }^{1}$, Pavel Doronkin ${ }^{1}$, Aleksander Priklonsky ${ }^{1}$, Igor Kabashkin ${ }^{2}$ \\ ${ }^{1} D$ un $D$ centrs \\ 7 Margrietas street, Riga, Latvia \\ e-mail:info@ddcentrs.lv \\ ${ }^{2}$ Transport and Telecommunication Institute \\ 1 Lomonosova street, Riga, LV-1019, Latvia \\ e-mail:kiv@tsi.lv
}

\begin{abstract}
In the petroleum, natural gas and petrochemical industries, great attention is being paid to safety, reliability and maintainability of equipment. There are a number of technologies to monitor, control, and maintain gas, oil, water, and sewer pipelines. The paper focuses on operational modal analysis (OMA) application for condition monitoring of operating pipelines. Special focus is on the topicality of OMA for definition of the dynamic features of the pipeline (frequencies and mode shapes) in operation. The research was conducted using two operating laboratory models imitated a part of the operating pipeline. The results of finite-element modeling, identification of pipe natural modes and its modification under the influence of virtual failure are discussed. The work considers the results of experimental research of dynamic behavior of the operating pipe models using one of OMA techniques and comparing dynamic properties with the modeled data. The study results demonstrate sensitivity of modal shape parameters to modification of operating pipeline technical state. Two strategies of pipeline repair - with continuously condition-based monitoring with proposed technology and without such monitoring, was discussed. Markov chain reliability models for each strategy were analyzed and reliability improvement factor for proposed technology of monitoring in compare with traditional one was evaluated. It is resumed about ability of operating pipeline condition monitoring by measuring dynamic deformations of the operating pipe and OMA techniques application for dynamic properties extraction.
\end{abstract}

Keywords: pipelines, reliability, damages detection, operational modal analysis, modelling of dynamic deformations

\section{Introduction}

Oil and gas operators face problems to keep pipeline safe and workable to avoid adverse effect on environment and to fulfil obligations of product delivery. In the petroleum, natural gas and petrochemical industries, great attention is being paid to safety, reliability and maintainability of equipment. In this respect, data on failures, failure mechanisms and maintenance related to these industrial facilities and its operations have become of increased importance.

There are a number of technologies to monitor, control, and maintain gas, oil, water, and sewer pipelines. Most of these technologies rely on some kind of communication networks to transfer data collected from inside and outside of the pipelines to the control stations. Different network architectures have been used to provide reliable communication in pipeline systems and attempted to support pipeline monitoring.

Nowadays, most of the pipeline monitoring systems are based on wired networks to connect and communicate with pipeline sensors. A number of problems can be noticed for the monitoring systems using wired networks (Jawhar, Mohamed, and Shuaib, 2007), (Mohamed and Jawhar, 2008).. Three major problems can be mentioned. Firstly, any damage in any part of the wires of the network may significantly degrade the performance of the pipeline monitoring system. Secondly, the physical security of such systems cannot be achieved when the pipeline expands on large areas. Lastly, since most of the pipelines that carry gas, oil, water, or sewer are located underground or in zones difficult to reach, it is hard to locate faults and repair them. This makes the process of maintaining a faulty network a very complex task.

Advancements in sensor technology have made possible the automated real-time monitoring of the health of the pipeline systems. A number of sensor network based technologies have been designed to monitor pipelines and to provide remote facilities to detect and report the positions of any leakage, damage, or corrosion (Murphy, Laffey, O'Flynn, Buckley and Barton, 2007), (Jin and Eydgahi, 2008), (Stoianov, Nachman, Madden and Tokmouline, (2007). One can notice, however, that these systems are passive in the way that they only report on incidents and do not contribute to their forecast. 
On the other side, a number of robotic agent based technologies to monitor pipelines and maintain them from damage has also been developed in literature ( Nassiraei, Kawamura, Ahrary, Mikuriya and Ishii, 2007), (Scholl, Kepplin, Berns and Dillmann, 2000). These technologies are designed to detect and locate any leakage, damage, or corrosion. While a huge number of robot based systems have been proposed that are manually controlled, a few number have considered semi-autonomous/autonomous solutions. In (Kim, Sharma, Boudriga and S. Iyengar, 2010) cost effective, scalable, customizable, and autonomous sensor-based system, called SPAMMS is proposed. It combines robot agent based technologies with sensing technologies for efficiently locating health related events and allows active and corrective monitoring and maintenance of the pipelines. SPAMMS integrates RFID systems with mobile sensors and autonomous robots. While the mobile sensor motion is based on the fluid transported by the pipeline, the fixed sensors provide event and mobile sensor location information and contribute efficiently to the study of health history of the pipeline.

The new approach was proposed for monitoring of most risky parts of operating pipeline, including those that are crossing the railroad or speedway or pass close to residential district. This approach is based on Operational Modal Analysis (OMA) of pipeline dynamic properties applying wide-band deformation sensors. Since the beginning of the 90's, OMA attracts attention of researchers for dynamic properties study of big civilian objects. OMA techniques allow determining the modal properties of a structure using the system output data only, that is, the dynamic signals of the stressed structure. The system responds to ambient excitation solely and there is no test excitation affecting the system. OMA use some key approaches for evaluation of modal properties, which are distinguished by the methods of data arrangement and processing. OMA application for pipeline condition monitoring is attractive for several reasons:

- the opportunity to receive the dynamic characteristics of the unlimited part of a pipeline;

- modal characteristics are linearized thanks to a wide range of accidental excitations;

- all or some parts of the measured degree of freedom can be used for reference purpose, which significantly increases method resolution and helps to segregate the paired and closely located modes;

- $\quad$ it is useful for the vibration-based health monitoring (Zhang, Brincker, Andersen, 2005);

- it can be used for the implementation of preventive/predictive maintenance for condition monitoring of operating pipelines oriented on increase reliability and optimization of the relationship between equipment ownership and operating profits by balancing cost of maintenance with cost of equipment failure, and associated production losses.

Widest OMA application was found for the approaches using conversion in the time domain: Natural Excitation Technique (NExT) (Chang and Pakzad, 2013), model of the general Auto-Regression Moving Average vector (ARMA V) (George and Jenkins, 1976), Stochastic Subspace-based methods (D'ohler, 2011), and approaches in frequency domain, like Frequency Domain Decomposition (FDD) (Brincker, Zhang and Andersen, 2001) or least-squares complex frequency-domain (LSCF) (Peetersa, Van der Auweraera, Guillaumeb and Leuridana, 2004). In this research, the authors considered use of one of OMA approaches - Enhanced Frequency Domain Distribution (EFDD) for evaluation of the condition of a section of an operating pipeline.

This article considers application of OMA technique for damage identification of the experimental model of a pipeline with the actuation by flow inside. Gas or flow streaming inside a pipe excites plenty of structural modes allowing determination of modal properties of surveyed pipeline part. Whereas accelerations typically characterize dynamic behavior of a structure, wall deformations of motionless pipe may be more effective for this purpose. Nowadays, most advanced pipelines already have fiber-optic systems for condition monitoring (Inaudi, Glisic, 2010) however, its frequency range is low and they are limited to detect small local damages as joint weld or corrosion spots that cause $50 \%$ of gas pipelines problems as well as other material damages. Being limited by low frequency range, they are not sensitive enough to many important ambient impacts as well. Unlike fiber optics, the film-type piezo-electric sensors distributed along a pipe provide measurement of pipe wall deformations in practically unlimited frequency range. The model of operating pipeline facilitated by above deformation measurement system was called smart pipe. The pipe equipped with sensitive deformation transducers works as an antenna that feels any wall deformations caused by streaming flow inside or soil distortion outside. By the way, smart pipe provides adequate dynamic data, required for modal analysis. 
The task of discussed research study was to verify the applicability of OMA for damage identification of smart pipe under operating conditions similar to natural ones. Practical application of modal analysis methods requires identification of natural modes of a structure in determined frequency range, therefore an experimental phase was preceded by mathematical modelling using the finite-element technique (FET).

\section{Modelling}

For modelling a pipe, the triangular parabolic elements evenly spread over the whole surface of the model were used as finite elements (FE). Analysis of the normal modes shape and frequency of oscillation was done in respect to the first 20 natural modes, the displacement being used as a parameter.

Table 1 presents the calculated parameters of the natural modes of a pipeline model and Figure 1 demonstrates principal mode types.

Table 1. Modal frequencies of ideal and "faulty" pipes

\begin{tabular}{|c|c|c|c|c|}
\hline \multirow[b]{2}{*}{$\begin{array}{l}\text { Mode } \\
\text { No }\end{array}$} & \multirow{2}{*}{ Mode id } & \multicolumn{3}{|c|}{ Frequency } \\
\hline & & $\begin{array}{l}\text { ideal pipe, } \\
\mathrm{Hz}\end{array}$ & $\begin{array}{l}\text { fault } \\
\text { pipe, } \mathrm{Hz}\end{array}$ & $\begin{array}{c}\text { Modify } \\
\text {-cation, } \\
\%\end{array}$ \\
\hline $1 a$ & \multirow{2}{*}{$1^{\text {st }}$ bending } & 36.19 & 35.76 & $1 \%$ \\
\hline $1 b$ & & 36.19 & 35.79 & $1 \%$ \\
\hline $2 a$ & \multirow{2}{*}{$2^{\text {nd }}$ bending } & 219.35 & 212.61 & $3 \%$ \\
\hline $2 b$ & & 219.38 & 214.10 & $2 \%$ \\
\hline 3 & $1^{\text {st }}$ torsion & 487.61 & 478.06 & $2 \%$ \\
\hline $4 a$ & \multirow{2}{*}{$3^{\text {rd }}$ bending } & 585.56 & 562.30 & $4 \%$ \\
\hline $4 b$ & & 585.67 & 567.75 & $3 \%$ \\
\hline 5 & $1^{\text {st }}$ longit. & 787.34 & 772.96 & $2 \%$ \\
\hline $6 a$ & \multirow{2}{*}{$4^{\text {th }}$ bending } & 1079.65 & 1063.49 & $1 \%$ \\
\hline $6 b$ & & 1079.84 & 1064.07 & $1 \%$ \\
\hline
\end{tabular}

\begin{tabular}{|c|c|c|c|c|}
\hline \multirow{2}{*}{$\begin{array}{l}\text { Mode } \\
\text { No }\end{array}$} & \multirow{2}{*}{ Mode id } & \multicolumn{3}{|c|}{ Frequency } \\
\hline & & $\begin{array}{l}\text { ideal pipe, } \\
\mathrm{Hz}\end{array}$ & $\begin{array}{l}\text { fault } \\
\text { pipe, } \mathrm{Hz}\end{array}$ & $\begin{array}{l}\text { Modify- } \\
\text { cation, } \%\end{array}$ \\
\hline $7 a$ & \multirow{2}{*}{$\begin{array}{c}1^{\text {nd }} \\
\text { shell }\end{array}$} & 1141.86 & 1128.12 & $1 \%$ \\
\hline $7 b$ & & 1141.99 & 1128.60 & $1 \%$ \\
\hline $8 a$ & \multirow{2}{*}{$2^{\text {rd }}$ shell } & 1149.15 & 1134.48 & $1 \%$ \\
\hline $8 b$ & & 1149.18 & 1134.59 & $1 \%$ \\
\hline $9 a$ & \multirow[t]{2}{*}{$3^{\text {th }}$ shell } & 1172.39 & 1159.15 & $1 \%$ \\
\hline $9 b$ & & 1172.45 & 1159.60 & $1 \%$ \\
\hline $10 a$ & \multirow[t]{2}{*}{$4^{\text {th }}$ shell } & 1229.38 & 1215.44 & $1 \%$ \\
\hline $10 b$ & & 1229.45 & 1215.55 & $1 \%$ \\
\hline $11 a$ & \multirow[t]{2}{*}{$5^{\text {th }}$ shell } & 1338.21 & 1325.35 & $1 \%$ \\
\hline $11 b$ & & 1338.34 & 1326.02 & $1 \%$ \\
\hline
\end{tabular}

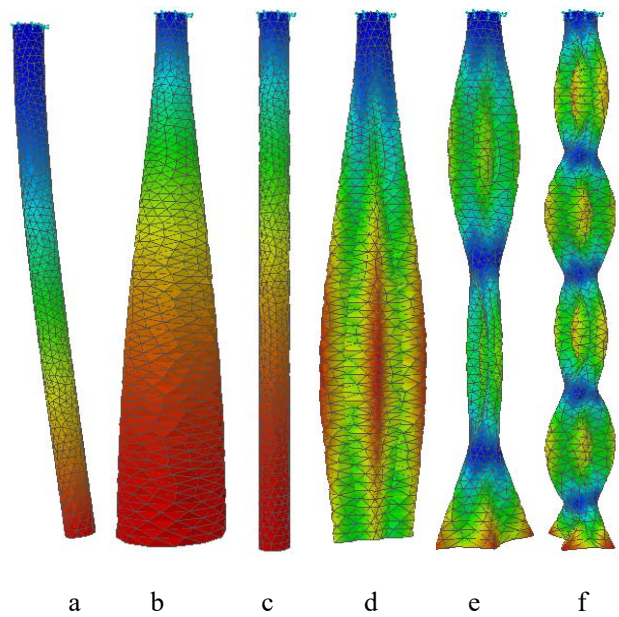

Figure 1. Calculated mode shapes

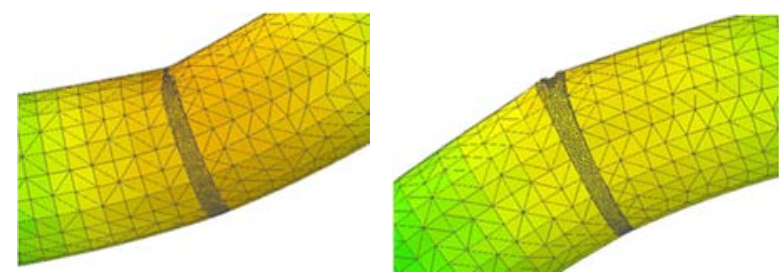

Figure 2. Mode modification after introducing a defect model

Column ideal pipe (Table 1) contains calculated frequencies of pipe model in ideal (non-faulted) state; column fault pipe includes frequencies of virtually damaged pipe. Analysis showed that the majority of the modes are paired, that is, they "sit" on the closely spaced frequencies and have similar modes with the $90^{\circ}$ phase shift. Paired modes have the same number but differ by letter $a$ or $b$. Symmetry of the pipe model cross section is the reason of the pairing. Identification of the modes allowed determining of two 
major mode groups and two isolated modes. The first group includes four pairs of bending modes: $1 a b$ (Figure 1a), 2ab, 4ab, 6ab, under which the pipe behavior is similar to bending oscillations of a beam. Such way of oscillation consider the cross sections of the hollow pipe deform in the same direction in which the longitudinal axis is bending. It means that at vertical bending mode the circular cross section of hollow pipe periodically compresses in vertical direction. Two isolated modes in low-frequency range are extremal cases of beam-like oscillation. The first one (No 3 in Table1, Figure 1b) is interpreted as the $1^{\text {st }}$ mode of torsion oscillations, in which the cross-sections of the pipe swivel around the longitudinal axis. The second one (No 5 in Table1, Figure 1c) is the longitudinal mode, in which the free end of the pipe shifts along the longitudinal axis. In both cases cross sections do not change its round shape but vary diameter.

The second modes group includes five pairs of oscillation modes: 7ab (Figure 1d), 8ab, 9ab (Figure 1e), 10ab, 11ab (Figure 1f), where the wall of the pipe behaves as a shell.

In each of the paired modes, the mode $b$ is similar to mode $a$, yet turned by $90^{\circ}$. Under shell oscillations the pipe model does not bend as a beam (the pipe axis is not deformed), however, the cross sections get deformed, being "flattened" and "stretched" in perpendicular directions.

To evaluate sensibility of natural modes to the local failure the computational experiment was done, where a defect of a real pipe was modelled as the pipe model wall saw-through (less than $10 \%$ of the circumference and $30 \%$ of the wall thickness).

Analysis of computed modes was limited by numeric evaluation of frequencies only, while evaluation of the mode shapes was done visually. However, since the frequency changing upon introduction of the defect is one of the modal parameters, it can be assumed that the mode is changing along with the change of frequency. Results of the computational experiment are shown in the column faulty pipe of Table 1. Different modes respond to the local fault in various ways. Thus, while the frequencies of the paired $1^{\text {st }}$ bending mode, which depends on the global properties of the modelled pipe, decreased by $1 \%$ only, the $2^{\text {nd }}$ and $3^{\text {rd }}$ modes that depend more on the mass and rigidity distribution along the model length decreased up to $4 \%$. For the shell modes, the modal frequencies decreased not more than $1 \%$. Varying response of the modes to a local fault is illustrated by Figure 2 showing the behavior of faulted area along the $3^{\text {rd }}$ bending mode (4ab Table 1) in the two opposite oscillation phases. As is evident, the defect coincides with the maximum deformation spot for this mode, therefore the parameters of that particular mode got distorted to the maximum extent.

Analysis of numerical pipe model identified two main groups of natural modes: bending and shell ones. Mode shapes of these groups have principal difference and their sensitivity to test fault differs.

\section{Experimental study of operating pipeline model by OMA technique}

The laboratory model of an operating pipeline was equipped with deformation sensors distributed along and circumferentially the pipe. The set of 21 deformation sensors provide data for experimental determination of the dynamic characteristics of a laboratory pipeline model. The laboratory pipeline model has the form of a straight section of a hollow pipe. Turbulent flow of water streaming through the pipe excites its walls. Controlled flow resistance in front of the pipe inlet allows adjusting of excitation level of the pipe walls by modulating the flow turbulence intensity.

Advanced piezoelectric film transducers (Figure 3) measure dynamic deformations of the oscillating pipe wall. Attached to the pipe surface the ultra-light sensors are exposed to stretching or compression together with the pipe wall almost without affecting the mechanical properties of the pipe. The preamplifier located next to the sensor on a flexible base transforms time-variant charge generated by the sensor under extension and compression of wall into the alternate electric voltage. Combination of the sensor and the preamplifier represents the deformation transducer.

Dynamic range of applied sensors is practically unlimited in terms of both the relative extension or compression and frequency. Tested segment of the experimental pipeline model has 21 transducers in the seven evenly distributed cross sections (Figure 4).

In each cross section, the transducers are located along the pipe axis on three lines that shifted at a $45^{\circ}$ angle from one to another. The transducers located on the top (blue line) and side (green line) generatrix are set by its length along the pipe axis (longitudinal transducers), and their task was to measure extension and compression of the "longitudinal" virtual fibers of the pipe. The transducers on the intermediary generatrix (red line) measured in circumferential direction that means deformation of pipe cross-section (lateral transducers). 
Multichannel data acquisition unit collects signals from all 21 transducers simultaneously and then transfers it to the computer that saves the data and performs OMA. The result of data processing is an eigenvector based on geometrical model of the pipeline, which includes the normalized deformation values describing shapes frequencies and damping factors of determined modes.

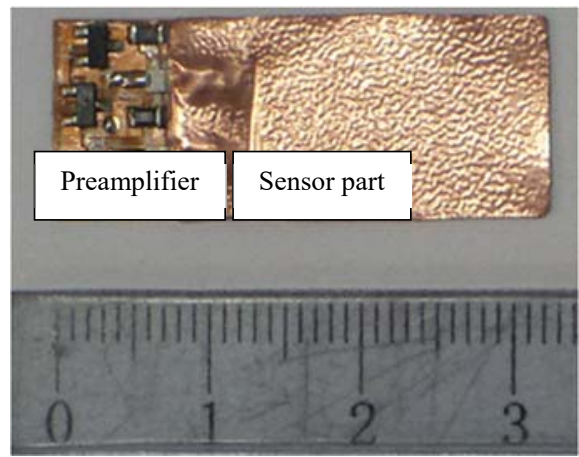

Figure 3. Deformation transducer

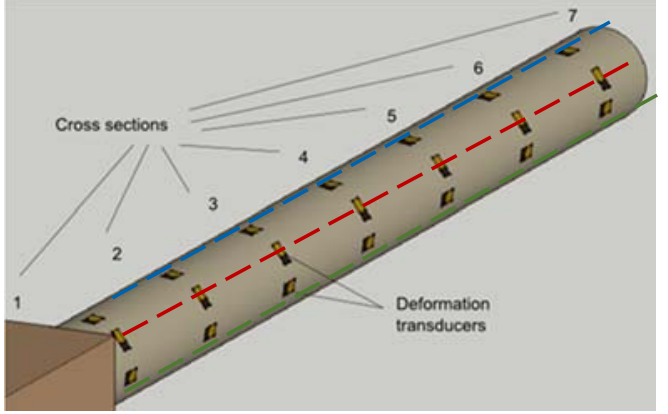

Figure 4. Distribution of transducers on the pipeline segment

\subsection{Mode shape identification of one-sided fixed pipe model}

The first version of operating pipeline model was built-up as one-sided fixed steel pipe with measurement set-up as on Figure 4. Diagrams, like presented on Figure 5, facilitate modes shape identification using experimental measured modal parameters. The normalized deformation magnitudes are related to the cross section number and plotted by taking into account the phase of oscillation (positive or negative). Depending on the location of the data points - the top and side of the pipe (longitudinal transducers) or between them (lateral transducers), the magnitude values on the diagram are connected by a blue, red or green line. Modes identification bases on the assumption that measured deformations of the pipeline model are linked through the second derivate with the displacements that were computed for numerical model of the pipeline. Figure 5a illustrates deformation of the pipe model oscillating at $1^{\text {st }}$ bending mode $(21,8 \mathrm{~Hz})$.

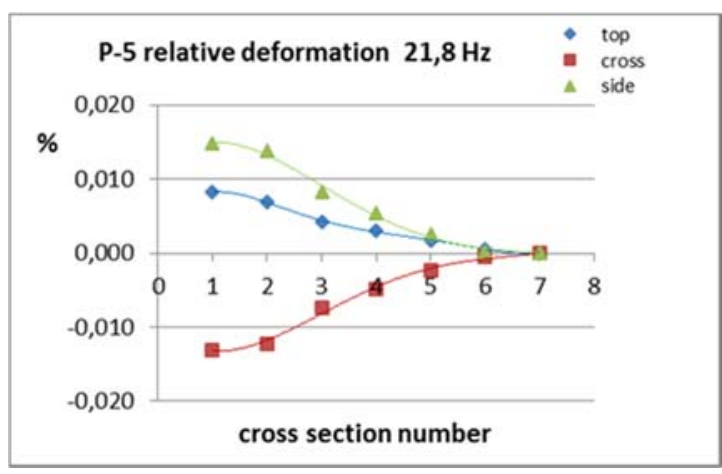

a)

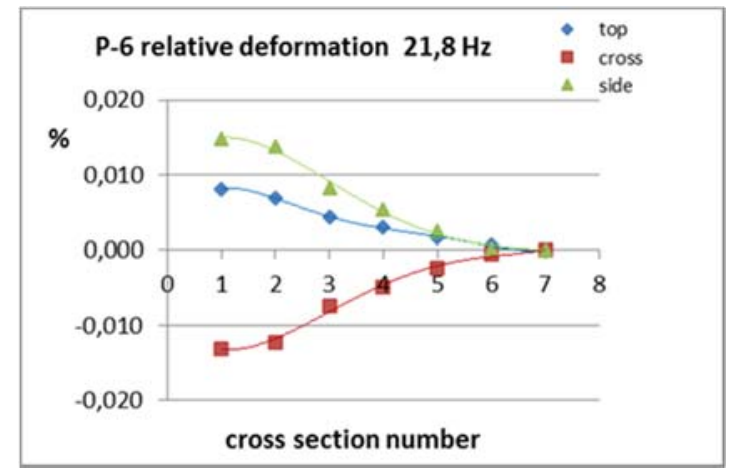

b)

Figure 5. Deformation diagrams of first bending mode: a) - initial condition, b) - faulted; top generatrix - blue rhombus; side - green cones; cross generatrix (lateral transducers) - red squads

The maximum displacement of the free end of the cantilever fitted pipe under the first bending mode is approaching zero deformation that may be seen in the section 7 for all transducers. Conversely, the minimum displacement next to pipe fixity $\left(1^{\text {st }}\right.$ section$)$ computed by numerical model is correspondent to the maximum deformation of the pipe walls. Comparison of deformation magnitudes along the top and the side generatrix in the same cross section allows determining the oscillation phase in relation to the fixed coordination system of the laboratory facility. The magnitudes and phase ratio of both lateral sensor lines 
give an idea about wall deformations distribution along the pipe model. A positive value of the top and the side deformation magnitudes indicates location of oscillation plane between its generatrix. Asymmetrical fixity of the operating pipe model causes $62^{\circ}$ slope of the oscillation plane (under the first bending mode) from the vertical that is why deformations at the side generatrix (green) dominate over the top generatrix (blue) deformations (Figure 5a). Bending of the pipe causes maximal "flattening" of cross sections near its fixity (cross section No 1), so side generatrix (red) appears to be located next to maximal compression line.

Analysis of the experimentally obtained modal shapes demonstrates their similarity to the numerically estimated data. At the same data numerically estimated modal frequencies appear to be lower than experimentally obtained due to simulation problems of experimental model boundary conditions. Thus, the first bending mode computed as $36 \mathrm{~Hz}$, in fact turned out $21.8 \mathrm{~Hz}$. Comparison of calculated and experimentally obtained mode shapes demonstrated their close resemblance, proving the model quality. In analyzing the properties of experimental model through the OMA technique no paired modes predicted by the digital model were revealed. That is because the fixity of the experimental pipe did not correspond to the numerical one.

To estimate the lowest sensitivity limit to mechanical properties modification the local damage was introduced into the laboratory pipeline model. Geometry of the fault was similar to the numerical model (paragraph 2), the cut-through the wall of the natural pipe was less than $30 \%$ of the wall thickness. Analysis of the experimentally obtained modal parameters of the pipeline model demonstrated variable response of different modes to modification of the technical state (as the numerical analysis also). The deformation distribution diagrams (OMA obtained) reflecting shapes of the first bending mode in the healthy (Figure 5a) and defective conditions (Figure 5b) did not differ practically. Integrated difference of normalized magnitudes between two states of the pipe did not exceed $0.2 \%$, while the frequencies differed by a mere $0.1 \%$, which corresponded to the estimated data.

At the same time, the differences proved more significant in some other modes. For example, the difference of magnitudes for the third bending mode oriented in the inclined plane between the initial (Figure 6a) and defective (Figure 6b) states was: in terms of magnitudes $-12.5 \%$, and in terms of frequency $-0.7 \%$. Modification of the mode shape happened primarily due to the change of the slope angle of the oscillation plane (in relation to the vertical) from $42^{\circ}$ to $25^{\circ}$ because of the defect. As we may see, the third bending mode has higher response to fault than the first one conditioned on the fact that location of the defect practically coincided with position of the maximum deformation for this mode, which was obvious already at the modelling stage.

Considered above means that mode shapes of higher order are more effective for detection of local failures of operating pipeline.

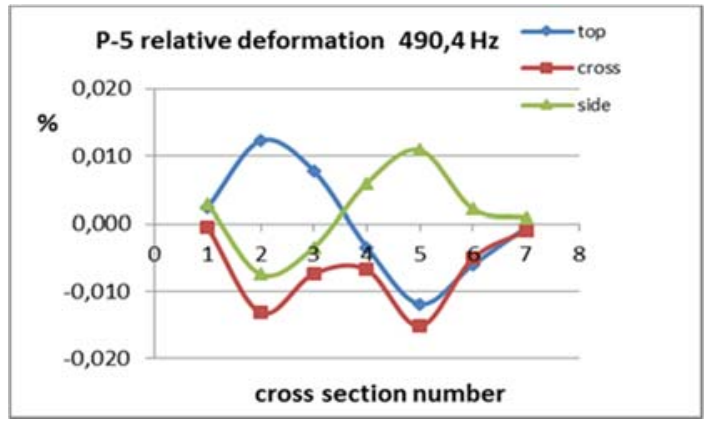

a)

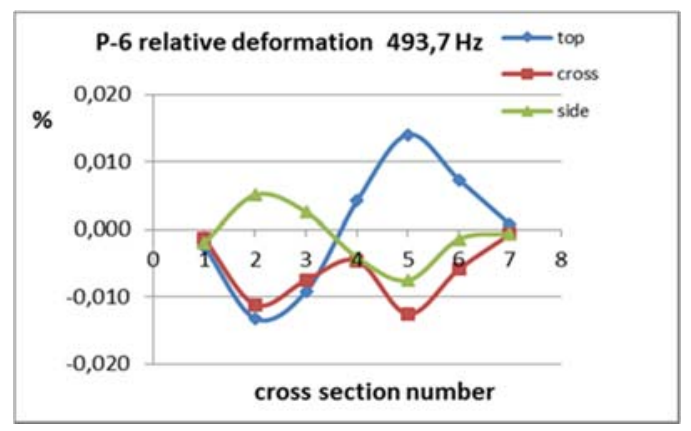

b)

Figure 6. Deformation diagrams of $3^{\text {rd }}$ bending mode: a) - initial condition, b) - faulted; top generatrix - blue rhombus; side - green cones; cross generatrix (lateral transducers) - red squads

\subsection{Impact of different media and faults to both-sided fixed pipe model}

Another operating pipeline model allowed to study its modal behavior in conditions similar to natural pipeline (Figure 7). 


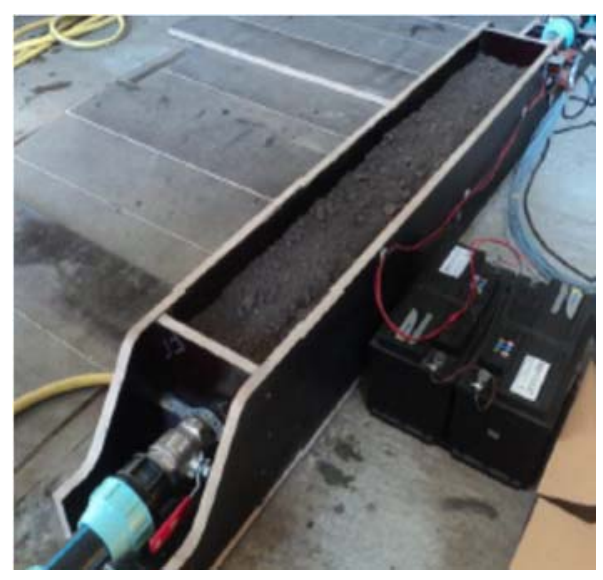

a)

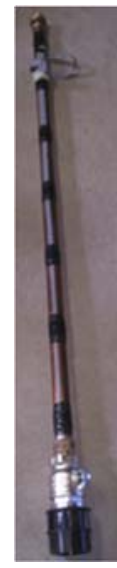

b)

Figure 7. Operating pipeline model: a) - testing box with pipe buried in the soil; b) - the pipe with transducers

Two opposite walls of the massive box (Figure 7a) are the pillars of the pipe with transducers (Figure 7b) fixing its ends in the transverse direction and limiting its movement in the axial direction. Both the box and the pipe model with the measurement system are waterproof. Ambient media in the box around the pipe model is variable: pipe can operate in open air, can be buried in a soil, and can be doused in a water. The copper-alloy pipe has the same measurement set-up as described in section 3.1.

As experimental data shows the properties of ambient media may seriously effect on modal parameters of the pipe model. Figure 8 illustrates how varying media modifies the shape of $3^{\text {rd }}$ shell mode of the pipe model in healthy state (no defects). It is evident that more consistent media reduce modal magnitudes slightly (Figure 8a, b, c). Media impact to modal frequency is more effective. For instance, loose soil reduced frequency or $3^{\text {rd }}$ bending mode for $0.2 \%$ only however, water dipping of the operating pipe reduced frequency of the same mode for almost $30 \%$. On top of media impact intensity and composition of the streaming flow may affect modal parameters of pipe model.

In case if media outside and flow inside remain stable in a determined time period, the pipe condition monitoring becomes available. For this purpose the parameter of modal parameters variation to be calculated.

The ensemble of estimated modal parameters experimentally obtained using OMA in the state $S$ of the pipeline model could be written as eigenvector matrix

$M_{S}=\left[m_{i, j, k}\right]$

where $i$ - number of DOF in one section (3), $j$ - number of sections (7), $k$ - mode number, $m_{i, j, k}-$ eigenvalue measured at $\operatorname{DOF}(i, j)$ of kth mode.

For monitoring purpose current state $S$ to be compared with initial state (etalon) $E$ by calculation of its difference

$\Delta M_{S}=M_{S}-M_{E}$

Modal parameters variation (MPV) estimates difference of modal parameters between current and initial state of the pipe model. Such differential eigenvector for current pipeline state estimates how far this current state of the pipe model is remote from its initial state (or etalon).

Experimental data obtained from testing of five technical states of the operating pipe model provided computation of MPV parameter for:

- healthy or initial state (three tests),

- local fault as thinning of the pipe wall (20\% of wall thickness and about $0.5 \%$ of square) made by polisher,

- $\quad$ static deflection of the pipe model (maximal deflection $-0,8 \%$ of the pipe model length) simulating earth-slide of natural pipeline.

Figure 9 illustrates variation of mode shapes and frequencies of operating pipe model at the $1^{\text {st }}$ bending mode in above mentioned states. 


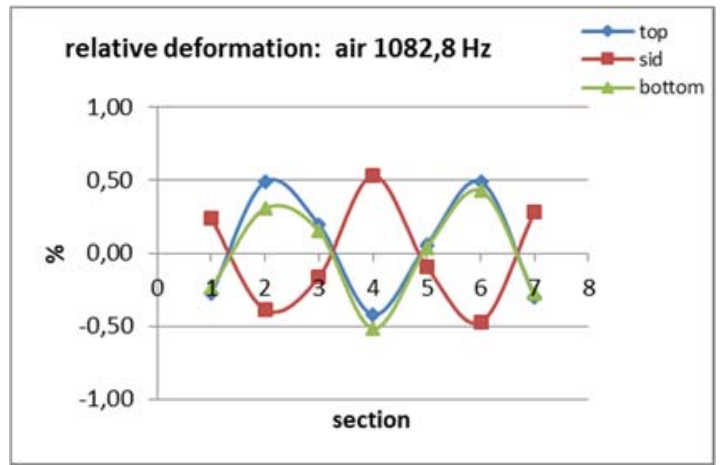

a)

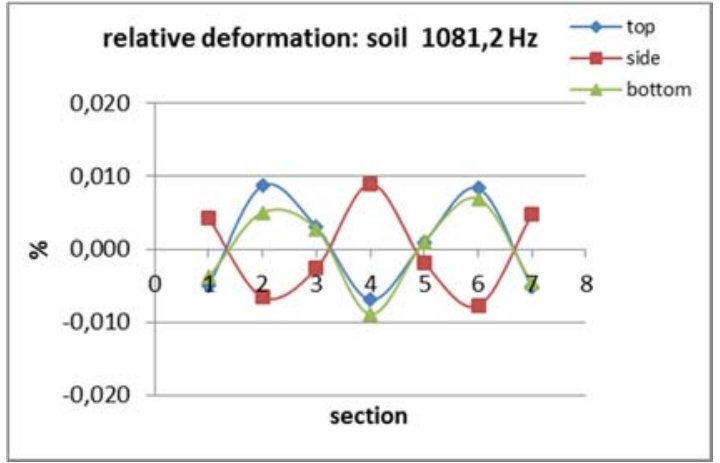

b)

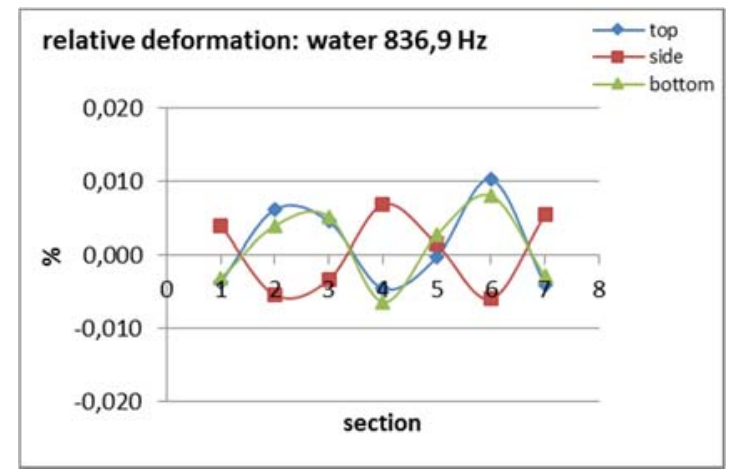

c)

Figure 8. Deformation diagrams of $3^{\text {rd }}$ shell mode with different media: a) - air, b) - soil, c) - water

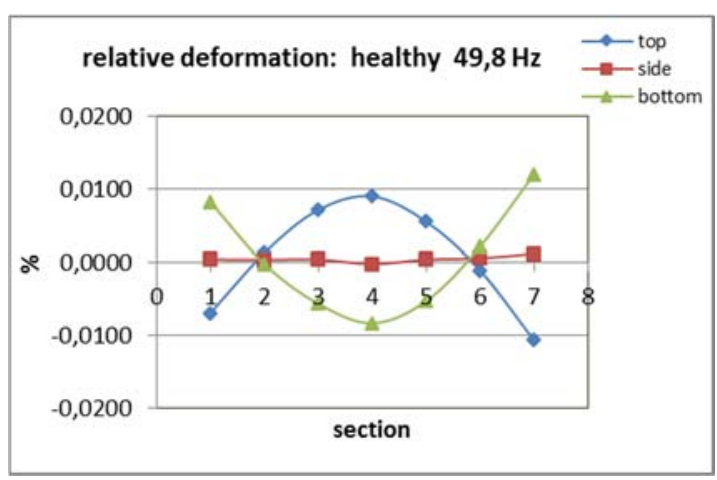

a)

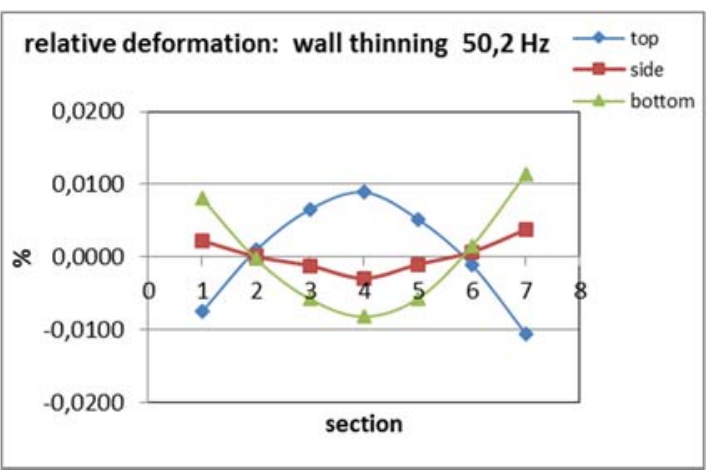

b)

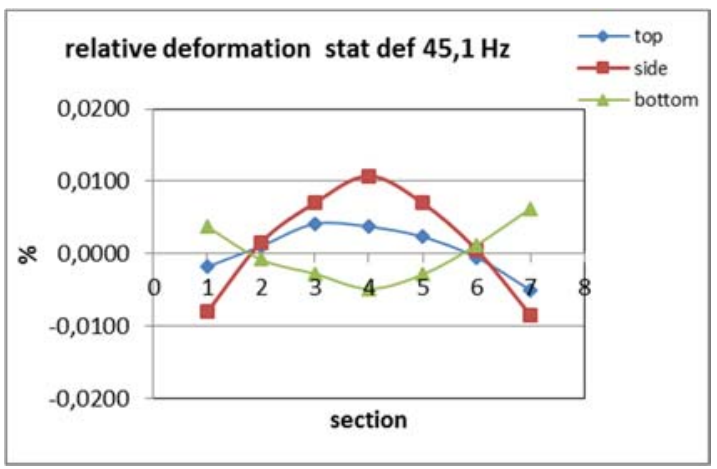

c)

Figure 9. Deformation diagrams of the $1^{\text {st }}$ bending mode in: a) - healthy state, b) - wall thinning, c) - static deflection 
Symmetry of diagram of healthy state (figure 9a) is broken by green line of side sensors line distortion (Figure 9b) when pipe wall was thinned locally. Maximal distortion of diagram red line locates close to the place, where the fault was implemented. Diagrams of Figure $9 \mathrm{c}$ dramatically change in comparison with healthy state (Figure 9a) as well as the frequency dropped for $10 \%$. The reason is that even slightly deflected pipe has lost symmetry and as the result has greatly modified its mechanical properties. Analysis of found mode shapes of the pipe model shows that MPV parameters have various sensitivity of bending and shell mode shapes to different faults. For instance, MPV of bending modes is more sensitive to static curve of the pipe than shell ones. However, sensitivity of MPV to local faults depends mostly of fault types and mode orders. It means, there is no privilege for MPV of mode types for detection of local faults.

As illustration of MPV sensitivity in depend of mode type Figure 10 shows MPV histogram for three pipe technical states. For both types of modes the scatter of MPV does not exceed 2.2..2.4dB within "healthy" states of operating pipe. Also in case of local fault (thinning) MPV for both modes grows to 4dB and more. However, static curve of operating pipe increase MPV of only bending modes (to 6.6dB), whereas shell modes parameter raises less (up to $4.3 \mathrm{~dB}$ ).

Results of OMA trial application for condition assessment of the experimental pipeline demonstrates varying sensitivity of the modal parameters to defects. While the frequencies of the natural modes changed less, modification of the pipe deformation shapes was quite obvious. That happened because the frequency of every mode is a global parameter of an object, while the deformation distribution (mode shapes) is the function of the local mass and stiffness distribution of the structure. Hence, the diagnostic efficiency of the modal shape parameters is much higher in comparison to the mode frequency parameters. This explains the fact that changes in the shape of the higher order modes as a response to a defect are able to become a reliable indicator of the alterations - even a relatively smaller-scale local changes of the structural state.

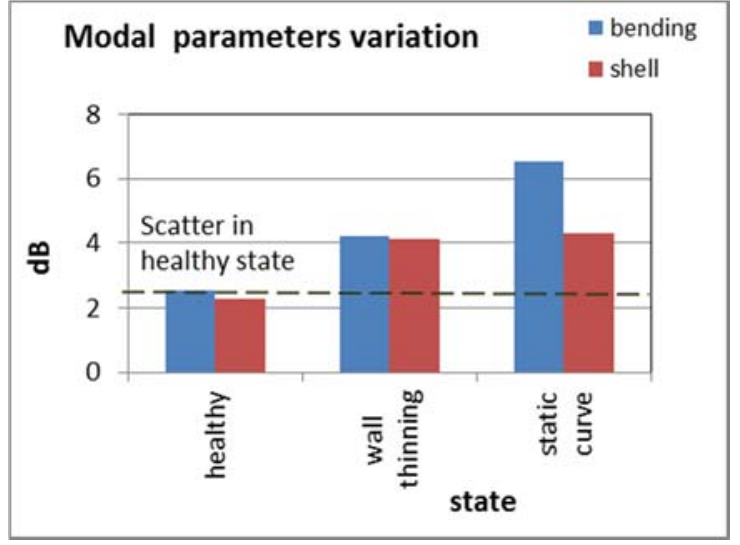

Figure 10. Modal parameters variation in depend of the pipe model technical state

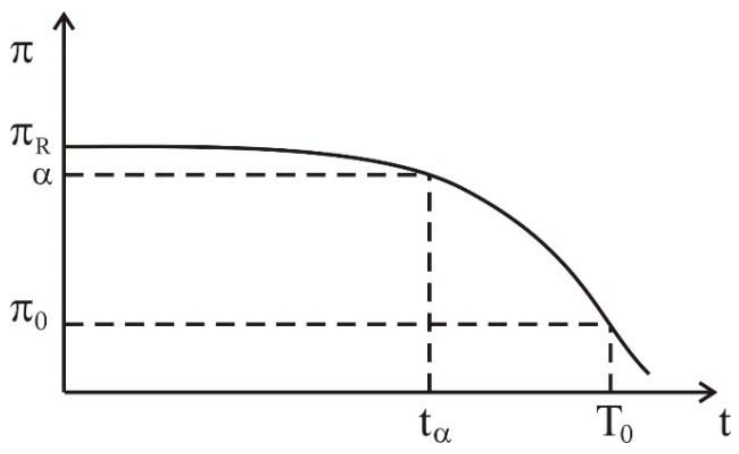

Figure 11. Deterioration of functional capabilities

\subsection{Operation modal analysis technique application on the natural scale operating}

To verify OMA applicability for monitoring purposes in actual environmental conditions the natural scale operating pipeline was built up (figure 12). The pipeline has total length more than $80 \mathrm{~m}, 88 \mathrm{~mm}$ diameter and pipe wall thickness $4 \mathrm{~mm}$. Pump station provides pressure of water up to 40Bar and flow stream velocity $1-5 \mathrm{~m} / \mathrm{s}$.

The pipeline is welded using separated steel pipes of $6 \mathrm{~m}$ length each. Above-ground pipeline part contains 5 pipes, under-ground one has two pipes. Pipelines are equipped by 168 deformation sensors (figure 3) allocated along seven tested pipes protected by cover layers. Tested pipes are connected by cables to measurement cabin where multichannel acquisition unit and PC are located. During the test the acquisition unit collects signals from transducers, provides its conditioning and transfers to PC that provides data collection and preliminary development. Operation modal analysis of collected data was applied at post processing stage. 


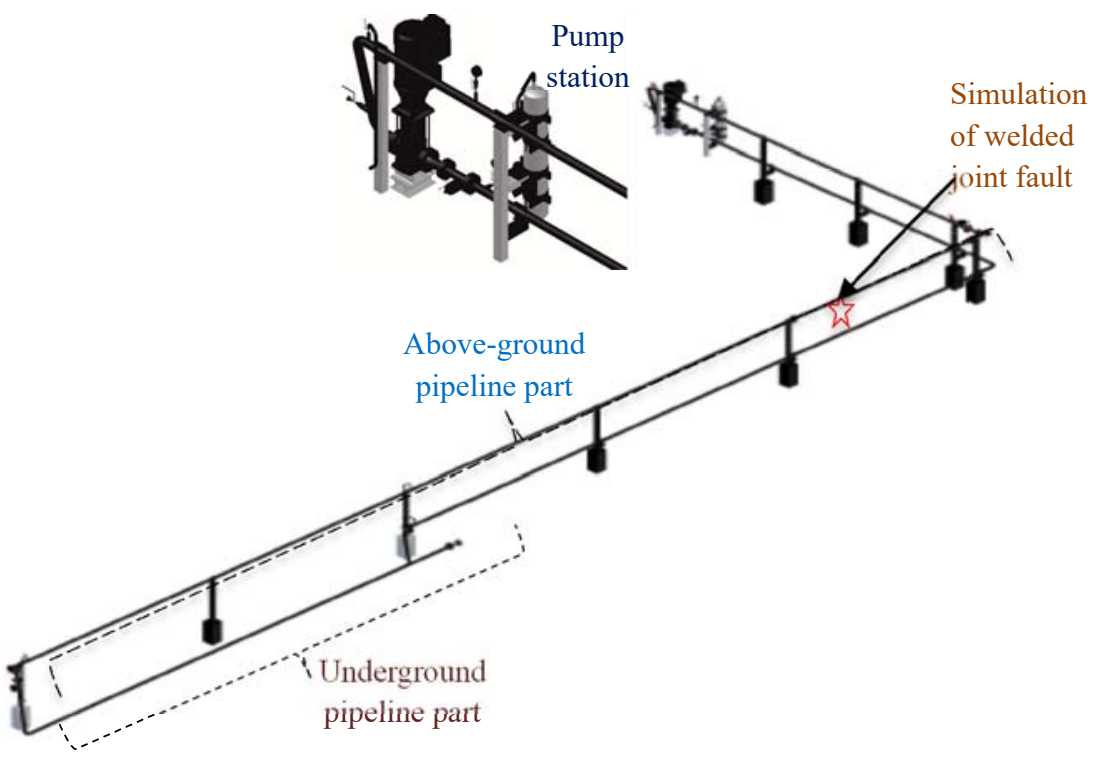

Figure 12. Natural scale operating pipeline model

The research study included test sessions when the pipeline operated at different modes (varied pressure and flow speed) at its healthy and faulty states. Test data development of healthy state pipeline confirmed as higher intensity of flow turbulence the better conditions for modal parameters identification using OMA technique. Simulation of welded joint fault (typical defect of operating pipelines) was implemented as the circumferential cut of one pipe wall. The cut was $1 \mathrm{~mm}$ depth and occupied $30 \%$ of pipe round. The fault was successfully identified using OMA that is illustrated by deformation diagrams on Figure 13.

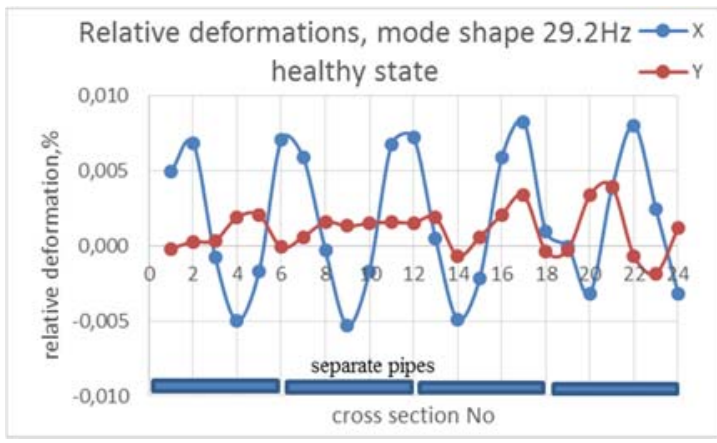

a)

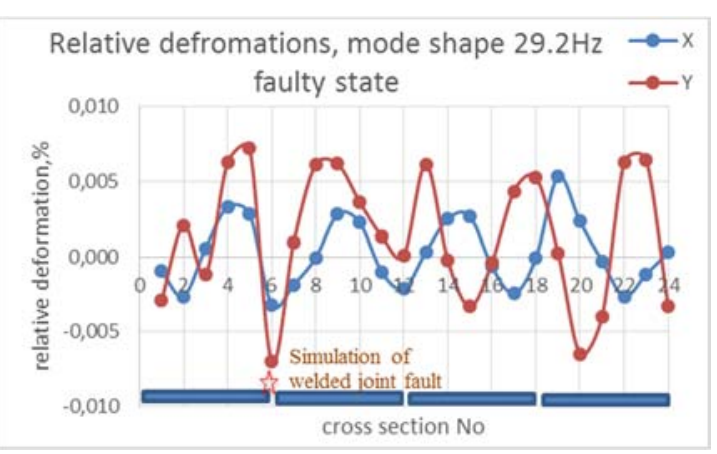

b)

Figure 13. Deformation diagrams of the $n^{\text {th }}$ bending mode of above-ground pipeline: a) - healthy state, b) - simulated fault of welded joint

Results of experimental study (including laboratory and natural scale pipelines tests) demonstrates two important outputs. From one hand, the flow streaming along a pipeline is able to generate its natural modes that could be identified using OMA techniques. From other hand, dynamic properties of pipe walls are sensitive enough to any damage that provides opportunity to detect state modification of operating pipe. These bring convincing proofs of OMA techniques capability to identify different damages of operating pipelines that allows application of actual condition based or predictive maintenance.

\section{Reliability of pipeline with OMA application for condition monitoring}

Oil and gas pipelines are critical infrastructure for effective transportation and distribution of energy resources. Pipelines can unexpectedly fail for many reasons including, corrosions, cracking, process upsets, 
and external environment. One of the key elements of maintainability is implementation of Preventive/Predictive Maintenance (PPM) based on condition monitoring of operating pipelines. Properly developed PPM programs are engineered efforts, which optimize the relationship between equipment ownership and operating profits by balancing cost of maintenance with cost of equipment failure, and associated production losses. Through the utilization of various nondestructive testing and measuring techniques, predictive maintenance determines equipment status before a breakdown occurs.

Proposed in this paper OMA technology of structural properties and dynamic deformations of pipeline can be an effective instrument for PPM. Let us investigate the impact of the adoption of technical condition diagnostics with OMA technology on the reliability of pipeline operation.

The typical deterioration of functional capabilities of pipeline shown at the Figure 11 (Guo, Song, Ghalambor, Lin, 2013). Condition-based monitoring (CBM) of parameter $\pi$ carry out with proposed technology with the sensitivity level $\alpha$. The moment $t_{\alpha}$ is point of time when you first detect incipient failure depends on the condition monitoring technique. If maintenance actions are not executed, the system failure occurs at time $T_{0}$ due to the development of degradation processes.

The following assumptions were made in their mathematical formulation:

- The service life of pipeline is infinite.

- The pipeline monitoring and maintenance system should be able to find any defects in the unhealthy pipeline under monitoring before any failure happens.

- Degradation rate for different modes of failure is constant (ISO 14224) with parameter $\lambda_{i}$, where $i=1, . . n$ is number of detected modes of failures.

- In the case of operation without CBM i-mode failure is hidden and continues to develop before the rise of system failure with the failure rate $\varphi_{i}, i=1, \ldots n$.

- When a failure detected during condition-based monitoring, a preventive maintenance is carried out with repair rate $\mu_{i}$, where $i=1, . . n$ is number of detected modes of failures.

- A pipeline after maintenance actions becomes as good as new.

- The occurrence of critical failures and their repair have an exponential distribution with parameters $\lambda_{0}$ and $\mu_{0}$ respectively.

Let us compare two strategies of pipeline repair - with continuously condition-based monitoring with proposed technology and without such monitoring. For this purpose, we use Markov chain model with discrete random process whose future states only rely on their current states and are independent of their past states.

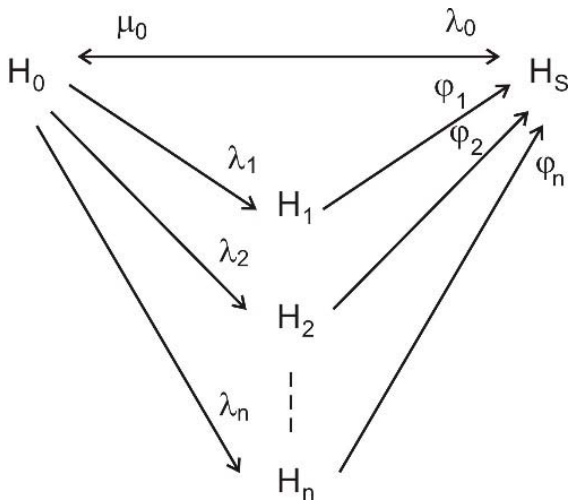

Figure 14. Markov's state transition diagram for system without condition-based monitoring

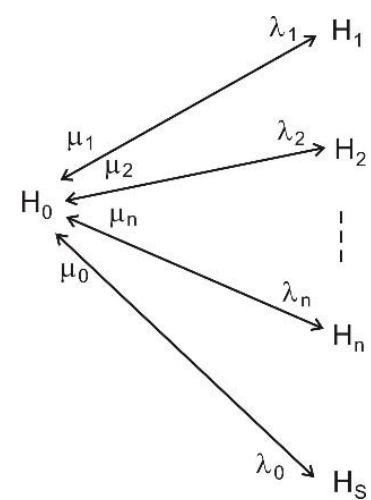

Figure 15. Markov's state transition diagram for system with continuously condition-based monitoring

Model 1. Strategy of pipeline repair without condition-based monitoring.

The behavior of the examined system is described by the state transitions (Figure 14): $H_{0}$ - state in which system is operating and available for use; $H_{i}$ - the appearance of i-mode failure $(i=1, \ldots n) ; H_{s}$ - the appearance of system failure.

On the base of the state transition diagram for a Markov's process shown in Figure 14, we can write the system of Chapman-Kolmogorov's equations: 


$$
\begin{aligned}
& P_{0}^{\prime}(t)=\mu_{0} P_{S}(t)-\left(\lambda_{s}+\sum_{i=1}^{n} \lambda_{i}\right) P_{0}(t) \\
& P_{i}^{\prime}(t)=\lambda_{i} P_{0}(t)-\varphi_{i} P_{S}(t), \quad i=\overline{1, n} \\
& P_{S}(t)=\lambda_{S} P_{0}(t)+\sum_{i=1}^{n} \varphi_{i} P_{i}(t)-\mu_{0} P_{S}(t)
\end{aligned}
$$

The normalizing condition is

$P_{S}(t)+\sum_{i=0}^{n} P_{i}(t)=1$

After transformation of the above mentioned set of equations we can obtain the value for $P_{i}(i=1, \ldots n, s)$ via $P_{0}$ :

$P_{i}=\frac{\lambda_{i}}{\varphi_{i}} P_{0}, \quad i=\overline{1, n}$

$P_{s}=\left(\lambda_{0}+\sum_{i=1}^{n} \varphi_{i}\right) \mu_{0}{ }^{-1} P_{0}$

Value of $P_{0}$ can be obtained by replacement $P_{i}(i=1 \ldots n)$ in the normalizing equation:

$P_{0}=\left[1+\left(\lambda_{0}+\sum_{i=1}^{n} \varphi_{i}\right) \mu_{0}^{-1}+\sum_{i=1}^{n} \frac{\lambda_{i}}{\varphi_{i}}\right]^{-1}$

Availability of system for this model is $A=P_{0}$.

Numerical values associated with the calculation of availability are often awkward for highly reliable systems. For this reason, it is more convenient to use the complement measure of availability, namely, unavailability U. Unavailability is the probability that an item will not operate correctly at a given time and under specified conditions (Dunn, William R, 2002). It opposes availability.

$$
\begin{aligned}
& U=1-A=P_{S}+\sum_{i=1}^{n} P_{i} \\
& U=\frac{a_{1}}{1+a_{1}} \\
& a_{1}=\left(\lambda_{0}+\sum_{i=1}^{n} \varphi_{i}\right) / \mu_{0}+\sum_{i=1}^{n} \lambda_{i} / \varphi_{i}
\end{aligned}
$$

Model 2. Strategy of pipeline repair with continuously condition-based monitoring.

The behavior of the examined system is described by the state transitions (Figure 15): $H_{0}$ - state in which system is operating and available for use; $H_{i}$ - the appearance of $\mathrm{i}$-mode failure $(\mathrm{i}=1, \ldots \mathrm{n})$ which is detected during condition-based monitoring, a preventive maintenance is started; $H_{s}$ - the appearance of system failure.

On the base of the state transition diagram for a Markov's process shown in Figure 14, we can write the system of Chapman-Kolmogorov's equations:

$$
\begin{aligned}
& P_{0}^{\prime}(t)=\sum_{i=1}^{n} \mu_{i} P_{i}(t)+\mu_{0} P_{S}(t)-\lambda_{0} P_{0}(t) \\
& P_{i}^{\prime}(t)=\lambda_{i} P_{0}(t)-\mu_{i} P_{i}(t), \quad i=\overline{1, n} \\
& P_{S}^{\prime}(t)=\lambda_{0} P_{0}(t)-\mu_{0} P_{S}(t)
\end{aligned}
$$


The normalizing condition is

$P_{S}(t)+\sum_{i=0}^{n} P_{i}(t)=1$

After transformation of the above mentioned set of equations we can obtain the value for $P_{i}(i=0, \ldots n, s)$ :

$P_{i}=\frac{\lambda_{i}}{\mu_{i}} P_{0}, \quad i=\overline{1, n}$

$P_{s}=\frac{\lambda_{0}}{\mu_{0}} P_{0}$

$P_{0}=\left(1+\sum_{i=0}^{n} \frac{\lambda_{i}}{\mu_{i}}\right)^{-1}$

Unavailability of system for this model is

$$
\begin{aligned}
& U=1-A=P_{s}+\sum_{i=1}^{n} P_{i} \\
& U=\frac{a_{2}}{1+a_{2}} \\
& a_{2}=\sum_{i=0}^{n} \frac{\lambda_{i}}{\mu_{i}}
\end{aligned}
$$

It is possible to evaluate the increasing of the reliability in the pipeline with continuously conditionbased monitoring in comparison with pipeline without such diagnosis with the help of the reliability improvement factor $V=U_{1} / U_{2}$, where $U_{1}$ - unavailability of the system in the first model and $U_{2}$ unavailability of the system in second one in accordance of equations (1) and (2).

Analysis of the coefficient $V$ indicates that the under the conditions $\lambda=k \varphi, \lambda=d \lambda_{0}, \mu=b \mu_{0}$ and $\lambda_{0}<<\mu_{0}$, that is done for most highly reliable systems, its value can be described by the approximate expression $V=z \gamma_{0}$, where $\gamma=\mu_{0} / \lambda_{0}, z=n k b /(n d+b)$.

Numerical example. At the Figure 16 there are $\mathrm{V}(\mathrm{b})$ dependencies for various failure modes $n$ and typical average meanings of the characteristics $k=1, M T B F=1 / \lambda_{0}=300$ days, $M T B R=1 / \mu_{0}=3$ days (Sahlqvist, 2014).

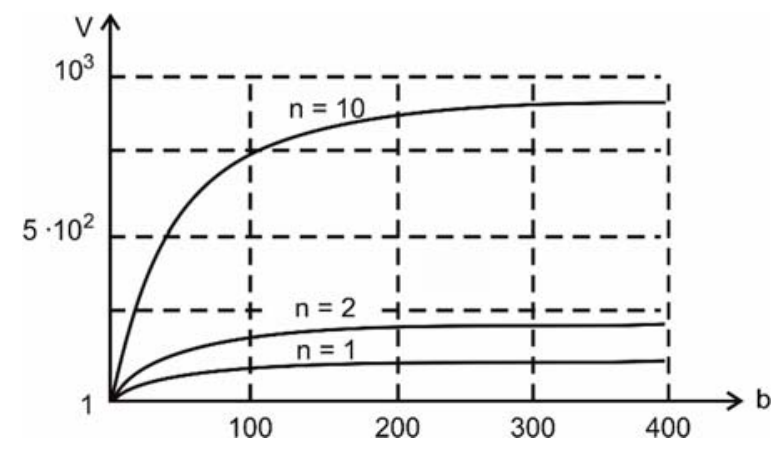

Figure 16. Variation of reliability deterioration coefficient

\section{Conclusions}

Finite-element modelling proved applicability of modal properties for condition monitoring of operating pipeline. Experimental model of operating pipeline of laboratory scale was built up for OMA techniques validation as the tool for pipe condition monitoring. Turbulent flow stream excited pipeline model walls provides ambient excitation required for OMA application. The measurement system including 
experimental piezo-electric deformation transducers and 21- channel acquisition unit characterized dynamic behavior of the pipeline functional model. The EFDD technique was applied to compute parameters of natural modes using experimentally obtained data. Numerically computed modes provide identification of experimentally obtained modes that ensures reliable estimation of the changes in the modal parameters. It was revealed that using the mode shape parameters as the diagnostic indicators is more advantageous compared to mode frequencies. It was proved that the modal parameters of the operating pipeline obtained using modal analysis technique are able to characterize its dynamic properties, so modification of these properties could be used as diagnostic indicators of pipeline model defects.

Two strategies of pipeline repair - with continuously condition-based monitoring with proposed technology and without such monitoring, was evaluated from reliability point of view. It is shown that reliability improvement factor for proposed method is by several orders of magnitude more effective in compare with traditional one. Thus, the research study of the pipeline model has proven ability of operating pipeline condition monitoring applying OMA techniques to dynamic deformations measured on the operating pipe.

\section{Acknowledgements}

The paper uses materials related to research study No. 1.22 of the project „Establishment of Transport Mechanical Engineering Competence Center" in cooperation with Investment and Development Agency of Latvia (L-KC-11-0002).

This work was supported by Latvian state research project "The Next Generation of Information and Communication Technologies (Next IT)" (2014-2017).

\section{References}

1. Jawhar, I.; Mohamed, N.; Shuaib, K. (2007) A Framework for Pipeline Infrastructure Monitoring Using Wireless Sensor Networks, in the Proceedings of the Sixth Annual Wireless Telecommunications Symposium (WTS 2007), Pomona, CA, USA, 26-28 April 2007; pp. 1-7.

2. Mohamed, N. and Jawhar, I. (2008) A Fault Tolerant Wired/Wireless Sensor Network Architecture for Monitoring Pipeline Infrastructures," in SENSOR-COMM '08: Proceedings of the 2008 Second International Conference on Sensor Technologies and Applications. Washington, DC, USA: IEEE Computer Society, pp. 179-184.

3. Murphy, F.; Laffey, D.; O’Flynn, B.; Buckley, J. and Barton, J. (2007) Development of a Wireless Sensor Network for Collaborative Agents to Treat Scale Formation in Oil Pipes, in EWSN, pp. 179-194.

4. Jin, Y. and Eydgahi, A. (2008) Monitoring of Distributed Pipeline Systems by Wireless Sensor Networks, in the Proceedings of the 2008 IJAC-IJME International Conference, Nashville, TN, USA.

5. Stoianov, I.; Nachman, L.; Madden, S. and Tokmouline, T. (2007) PIPENET: A Wireless Sensor Network for Pipeline Monitoring, in the Proceedings of the 6th international conference on Information processing in sensor networks. New York, NY, USA: ACM, pp. 264-273.

6. Nassiraei, A.; Kawamura, Y.; Ahrary, A.; Mikuriya, Y. and Ishii, K. (2007) Concept and Design of a Fully Autonomous Sewer Pipe Inspection Mobile Robot Kantaro, in the Proceedings IEEE International Conference of Robotics and Automation, April 2007, pp. 136-143.

7. Scholl, K.-U.; Kepplin, V.; Berns, K. and Dillmann, R. (2000) Controlling a Multi-Joint Robot for Autonomous Sewer Inspection, in the Proccedings of the IEEE International Conference on Robotics and Automation ICRA '00, vol. 2, pp. 1701-1706.

8. Kim, J.-H.; Sharma, G.; Boudriga, N. and Iyengar, S. (2010) SPAMMS: A Sensor-Based Pipeline Autonomous Monitoring and Maintenance System, in the Proceedings of the Second International Conference on Communication Systems and Networks (COMSNETS), 5-9 Jan. 2010, pp.1-10.

9. Minwoo Chang and Shamim N. Pakzad. (2013) Modified Natural Excitation Technique for Stochastic Modal Identification. Journal of Structural Engineering, ASCE, October, pp. 1753-1762.

10. George, E. P. Box, Gwilym M. Jenkins. (1976) Time series analysis: forecasting and control. San Francisco. Holden-Day.

11. D'ohler, M. (2011) Subspace-Based System Identification and Fault Detection: Algorithms for Large Systems and Application to Structural Vibration Analysis. Dynamical Systems. Universit'e Rennes Nr.1.

12. Brincker, R.; Zhang, L. and Andersen, P. (2001) Modal Identification of Output-Only Systems Using Frequency Domain Decomposition. Smart Materials and Structures, 10 (2001) 441-445. 
13. Bart Peetersa, Herman Van der Auweraera, Patrick Guillaumeb and Jan Leuridana. (2004) The PolyMAX Frequency-Domain Method: A New Standard for Modal Parameter Estimation? Shock and Vibration. T. 11. - №. 3-4. - C. 395-409.

14. Zhang, L.; Brincker, R. and Andersen, P. (2005) An Overview of Operational Modal Analysis: Major Development and Issues, in the Proceedings of the 1st International Operational Modal Analysis Conference, Copenhagen, Denmark.

15. Inaudi, D.; Glisic, B. (2010) Long-Range Pipeline Monitorin, 400 p. 